Mon. Not. R. Astron. Soc. 000, 1-4 (2005) ～Printed 3 January $2019 \quad$ (MN LTEX style file v2.2)

\title{
Strange Pulsar Hypothesis
}

\author{
Raka Dona Ray Mandal $^{1}$, Monika Sinha ${ }^{1 *}$, Manjari Bagchi ${ }^{1}$, Sushan Konar ${ }^{2}$, Mira Dey ${ }^{1 * *}$ \\ and Jishnu Dey ${ }^{1}$ \\ 1 Physics, Presidency College, Kolkata, India \\ ${ }^{2}$ CTS \& Physics, Indian Institute of Technology, Kharagpur, India \\ * Research Fellow, CSIR, GOI \\ ** Emeritus Scientist, CSIR, GOI \\ e-mail : rakadona@vsnl.net, monika2003@vsnl.net,mnj2003@vsnl.net,sushan@iitkgp.ac.in,deyjm@giascl01.vsnl.net.in
}

\begin{abstract}
It appears that there is a genuine shortage of radio pulsars with surface magnetic fields significantly smaller than $\sim 10^{8}$ Gauss. We propose that the pulsars with very low magnetic fields are actually strange stars locked in a state of minimum free energy and therefore at a limiting value of the magnetic field which can not be lowered by the system spontaneously.
\end{abstract}

Key words: superconductivity-magnetic fields-stars : strange-pulsars-general

\section{INTRODUCTION}

A radio pulsar is a strongly magnetized rotating neutron star. The dipolar component of the magnetic field can be estimated from its spin-period $(P)$ and the period derivative $(\dot{P})$, apart from some structural constants $(\mathcal{B} \propto \sqrt{P \dot{P}})$. Magnetic fields estimated in this fashion broadly classifies the radio pulsars in two categories a) isolated pulsars with rotation periods usually above $1 \mathrm{~s}$ and very strong magnetic fields $\left(10^{11}-10^{13} \mathrm{G}\right)$; (b) binary/millisecond pulsars with much shorter rotation periods and considerably weaker magnetic fields $\left(10^{8}-10^{10} \mathrm{G}\right)$. Observations suggest a connection between the low magnetic field of the second group with their being processed in binary systems, indicating an accretion-induced field decay in such cases (see Bhattacharya (2002) for a review).

However, none of the existing mechanisms of field evolution implies that the final field should saturate to a particular value irrespective of the evolutionary history of a given pulsar. Surprisingly, the observed pulsar population seems to have a lower bound for the magnetic field strength. Till date some fifteen-hundred radio pulsars have been discovered, for a large number of which the values of $P$ and $\dot{P}$ (and therefore $\mathcal{B}$ ) are available. Fig.[1] shows a plot of the magnetic field vs. the spin period $(\mathcal{B}-P)$ of these pulsars. It appears that there exist a minimum value of the magnetic field, around $\sim 10^{8} \mathrm{G}$.

In the present work, we try to offer an explanation for the minimum magnetic field by associating the millisecond pulsars with the strange stars. The relation between the decay of the magnetic field and the binary history of a pulsar has been firmly established. It is also understood that the very low-field pulsars, in particular the millisecond pulsars, are products of low-mass X-ray binary systems (LMXB). Recently, there has been an attempt to associate the bottom magnetic field of the millisecond pulsars with the total amount of mass accreted (Zhang 2004). However, we feel that the minimum field, characteristic of pulsars processed in LMXBs, arises due to an entirely different reason. Because of the long evolutionary timescales the total amount of mass accreted by a neutron stars in an LMXB is quite large. Addition of this extra mass may trigger the conversion of a neutron star into a strange star via deconfinement (Olinto 1987). Interestingly, many of the compact objects suspected to be strange stars are residing in LMXBs (Li et al. 1999; Datta, Thampan, \& Bombaci 2000). In view of this, we suggest that it is the intrinsic property of a strange star which gives rise to a lowerbound of the magnetic field.

Strange Quark Matter (SQM), composed of u, d and s quarks, may probably be the ultimate ground state of matter (Witten 1984). It has been found that the stable range of mass $\left(1 \mathrm{M}_{\odot}-2 \mathrm{M}_{\odot}\right)$ for strange stars is quite similar to that for neutron stars. Furthermore, in this range the radii of strange stars are not very different from those of the standard neutron stars (Haensel, Zdunik, \& Schaefer 1986). Since the range of stable rotation periods sustainable by these two types of stars are also similar, it has always been suspected that some of the pulsars could very well be strange stars (Alcock, Farhi, \& Olinto 1986). In this article we take the view that if not all pulsars then at least the millisecond pulsars could be strange stars.

The aim of the present work is to show that the physics of strange stars naturally gives rise to a lower limit of the magnetic field. In fact, a tuning of the QCD parameters does produce a value very close to $10^{8}$ Gauss. The paper is organized as follows. In section 2 we discuss our model and describe the calculations in section 3 . And finally, we present our conclusions in section 4. 


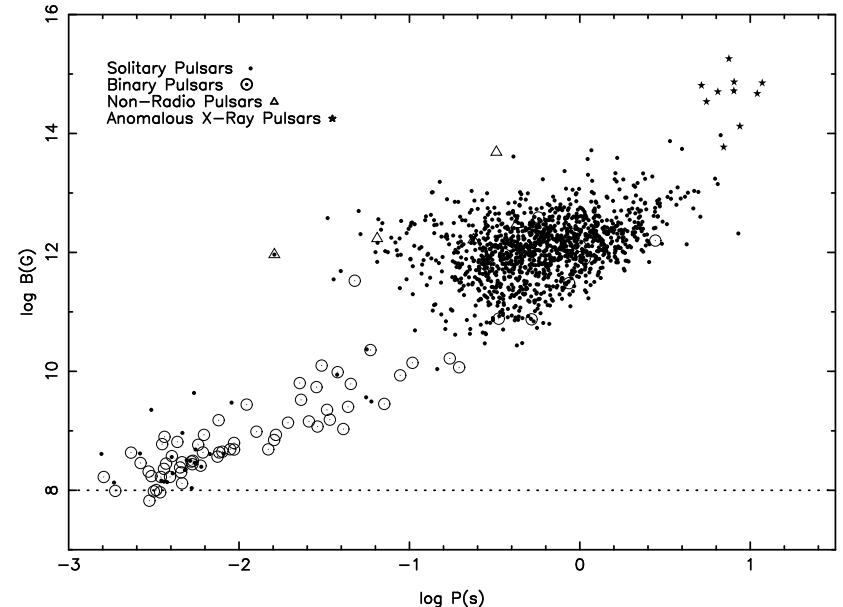

Figure 1. Magnetic field vs. spin-period of observed pulsars. The data is obtained from the ATNF on-line pulsar catalog available at http://www.atnf.csiro.au/research/pulsar/psrcat/ and include both galactic and extra-galactic pulsars.

\section{DI-QUARK FORMATION}

To explain the observed minimum of the pulsar magnetic field we consider a rotating strange star model. The strange star is understood to consist of a $u d s$ plasma with a small admixture of electrons to maintain overall charge neutrality, where each particle species is Fermi-degenerate (Witten 1984; Benvenuto, Horvath, \& Vucetich 1991; Benvenuto, Vucetich, \& Horvath 1991). The star may also support a thin hadronic crust of mass $M_{\text {crust }} \lesssim 10^{-5} \mathrm{M}_{\odot}$ (Alcock, Farhi, \& Olinto 1986). In our discussion we shall neglect the effect of this crust.

In this work, we construct a rotating strange star using the recently proposed realistic quark matter EOS in which - (a) a mean field is derived from a two-body potential incorporating asymptotic freedom with a deconfinement transition and (b) a density dependent ansatz for the quark mass using chiral symmetry restoration at high density (Dey et al. 1998). This EOS produces an absolutely stable SQM with the same parameters for which $u d$ matter is unbound. The surface of such a star is rather sharp, since the deconfinement transition for strong interaction sets in very quickly at a critical density of about $\sim 4.5 \rho_{0}$, where $\rho_{0}=0.17 / \mathrm{fm}^{3}$. This model has been successfully used to describe rotating strange stars (GondekRosińska et al. 2000; Bombaci, Thampan, \& Datta 2000).

Apart from the mean field there exists a spin-dependent part of the interaction responsible, for example, for the $\pi-\rho$ or the $N-\Delta$ mass splitting. This interaction potential, allowing the quarks to interact with each other to form di-quarks in definite color-spin channel, is given by (Sinha et al. 2002),

$V_{i j}=-V_{0}\left(\lambda_{i} \cdot \lambda_{j}\right)\left(S_{i} . S_{j}\right) e^{-\sigma^{2} r_{i j}^{2}}$

where $V_{0}$ is the strength and $\sigma$ is the range of the potential. $\lambda$ and $S$ are the colour and spin matrices and $r_{i j}$ is the distance between the $i$-th and the $j$-th particle. $V_{0}$ and $\sigma$ are adjusted such that the predictions for $\pi-\rho$ or $N-\Delta$ splitting are well within the experimental error limits. This interaction is assumed to be instanton induced with a constant strength through the entire density range.
There can be two types of di-quarks - flavor symmetric and antisymmetric. With the negative sign in Eq.(1) the potential is attractive in two combinations - (i) the spin singlet, colour antisymmetric state $(\overline{3})$ and (ii) the spin triplet, colour symmetric state (6). With such spin-color combinations, $u d$ pairs formed in the flavor antisymmetric state with $L=0$ and ss pairs formed in the $L=1$ state will decrease the energy further (Sinha et al. 2005). Since the pairs are not physically bound they would experience stronger attraction when they come closer but their kinetic energy may overcome the potential and take them apart again. However, on the average there would be a fixed number of charged di-quarks at any instant. Bailin \& Love (1984) have shown that this can give rise to superconductivity within the star even when the momentum transfer is large.

In a rotating star with one superconducting species, a uniform magnetic field (London field) is set up such that,

$B=\frac{2 m^{*} c}{q^{*}} \Omega$

where $m^{*}$ and $q^{*}$ are the effective mass and the charge of the pair. $\Omega$ is the angular velocity of the star (Baym 1988). Typically the strength of the London field is rather small and is $\sim 10^{-1} \mathrm{G}$ for the $u d$ pair.

However, the situation is much more complicated with two species of di-quarks. In a rotating strange star both the $u d$ and the $s s$ superconducting pairs are present. The London field would be different for pairs with different mass and charge. From the minimization of the Ginzburg-Landau free energy Chau (1997) concluded that at least one species will rotate without creating vortices, when the species are non-interacting. The other species, having a different London field, would create vortices to rotate with the same angular velocity. But if the species are interacting then they would form vortex bundles surrounding a common normal core with a magnetic flux trapped within. We look at the minimization of GinzburgLandau free energy density of such a system to estimate the magnetic field of such a star.

\section{MINIMUM ENERGY STATE}

To calculate the Ginzburg-Landau free energy of a rotating strange star, consisting of $u d$ and $s s$ superconducting pairs, we assume the pair momenta are small and the use of non-relativistic GinzburgLandau theory is appropriate. Moreover, as mentioned above, we expect them to exhibit superfluidity. A superfluid supports rotation by forming a number of Onsager-Feynman vortices each carrying a fixed quantum of circulation inversely proportional to the effective mass.

For a star rotating with an angular velocity $\Omega$, the Ginzburg-Landau free energy functional of a di-quark assembly, is given by (Sauls 1989; Chau 1997), $F=\int f d V$, where the energy density $f$ consists of - (a) the magnetic energy of the superconductor, (b) the kinetic and the nucleation energy of the vortices and (c) the interaction energy between different quark species if appropriate. The order parameter for the $j$-th species is $\Psi_{j}(\mathbf{r})\left(=\left|\Psi_{j}\right| e^{i \phi_{j}(\mathbf{r})}\right)$ where $\phi_{j}$ is the phase and $r$ is the distance variable. Then, in the co-rotating frame, the Ginzburg-Landau free energy density for the $j$-th species can be written as, 
$f_{j}=-a_{j}\left|\Psi_{j}\right|^{2}+\frac{b_{j}}{2}\left|\Psi_{j}\right|^{4}+\frac{1}{2 m_{j}^{*}}\left|\mathcal{P}_{j} \Psi_{j}\right|^{2}+\frac{|\nabla \times \mathbf{A}|^{2}}{8 \pi}$

where $a$ and $b$ are positive constants such that the superconducting state is preferred over the normal state and $m_{j}^{*}$ is the effective mass of the $j$-th pair. $\mathbf{A}$ is the corresponding vector potential satisfying $\mathbf{B}=\nabla \times \mathbf{A}$. Because of the rotation a uniform magnetic field is set up such that $\mathbf{A}=(\mathbf{B} \times \mathbf{r}) / 2$ along an array of vortices in the superconducting interior of the star. The kinetic energy of the $j$-th species is determined by its momentum operator $\mathcal{P}_{j}$, given by,

$\mathcal{P}_{j}=-i \hbar \nabla_{j}+\frac{q_{j}^{*}}{c} \mathbf{A}-m_{j}^{*}\left(\boldsymbol{\Omega} \times \mathbf{r}_{j}\right)$,

where $q_{j}^{*}$ is the effective charge of the pair. If the quark species are non-interacting then the above-mentioned free energy takes the following form (Chau 1997):

$$
\begin{aligned}
f= & \frac{\mathcal{B}^{2}}{8 \pi} \\
& +\sum_{j}\left\{\frac{\hbar \rho_{s j}}{m_{j}^{*}}\left[\ln \left(\frac{R_{c j}}{\xi_{j}}\right)-\frac{3}{4}\right]+m_{j}^{*} \frac{\xi_{j}^{2}}{2 \hbar} N_{j}(0) \Delta_{j}^{2}\right\} \\
& \times\left|\Omega-\frac{q^{*}}{2 m_{j}^{*} c} \mathcal{B}\right|
\end{aligned}
$$

where, $\rho_{s j}$ is the density of the superconducting species, $R_{c j}$ is the inter-vortex spacing, $\xi_{j}$ is the coherence length or the radius of the core of the vortex and $\frac{1}{2} N(0) \Delta^{2}$ is the difference in the energy density between the normal and the super-conducting phase. The vortices of each of the non-interacting di-quark species can form its own Abrikosov lattice. However, the structure of $f$ is such that there exists a situation where only one species of superconducting di-quarks form vortices whereas the other species rotate without creating vortices.

However, we consider the more realistic situation in which the superconducting di-quark species are interacting. The interaction induces a drag energy. To minimize this drag a vortex bundle is created where all the species share common normal core of radius $\xi$. Assuming the velocities of all the species to be the same, the vortex bundle density $\mathcal{D}$ is given by :

$\mathcal{D}=2 \Omega / K$

where $K$ is the circulation constant defined as,

$K=\frac{h}{m_{s}^{*} q_{u d}^{*}-m_{u d}^{*} q_{s}^{*}}\left(q_{u d}^{*} N_{s}-q_{s}^{*} N_{u d}\right)$

with $N_{j}$ being the number of vortex quantum per bundle. Then, inclusion of interaction changes the free energy functional to the following,

$$
\begin{aligned}
f= & \frac{\mathcal{B}^{2}}{8 \pi} \\
+ & {\left[\sum_{j}\left\{\frac{h^{2} \rho_{s j} N_{j}^{2}}{4 \pi m_{j}^{* 2}}\left[\ln \left(\frac{R_{c j}}{\xi_{j}}\right)-\frac{3}{4}\right]+\frac{\pi \xi_{j}^{2}}{2} N_{j}(0) \Delta_{j}^{2}\right\}\right.} \\
& \left.+\frac{\Phi_{v}^{2}}{8 \pi^{2} \lambda^{2}}\right] \times|\mathcal{D}|
\end{aligned}
$$

where $\Phi_{v}$ is the magnetic flux in the core of a vortex bundle given by,

$$
\Phi_{v}=\frac{h c}{m_{s}^{*} q_{u d}^{*}-m_{u d}^{*} q_{s}^{*}}\left(m_{u d}^{*} N_{s}-m_{s}^{*} N_{u d}\right) .
$$

\section{MINIMUM MAGNETIC FIELD}

Typically, we expect the system to favour the minimum energy configuration. As can be seen from the form of the free energy functional, its minimization essentially puts a lower bound on the magnetic field. Therefore, once the system gets locked into this minimum energy state, there is no natural way of decreasing the magnetic field further.

For the ground state configuration $N_{u d}=0$ and $N_{s}=1$, giving

$$
K=\frac{h q^{*} u d}{m_{s}^{*} q_{u d}^{*}-m_{u d}^{*} q_{s}^{*}}, \quad \Phi_{v}=\frac{h c m_{u d}^{*}}{m_{s}^{*} q_{u d}^{*}-m_{u d}^{*} q_{s}^{*}}
$$

Although there are no vortex quanta for the $u d$-pair both the mass and the charge of this pair enter in $K$ as well as in $\Phi_{v}$. This happens because of the strongly interacting nature of the system.

To estimate the minimum field we use the following parameter values. The masses of the di-quark pairs are taken to be $m_{u d}^{*} \sim$ $270 \mathrm{MeV}$ and $m_{s}^{*} \sim 560 \mathrm{MeV}$ (Sinha et al. 2002). With these we have, $\mathcal{D}=3.7 \times 10^{3} \nu \mathrm{cm}^{-2}$ and $\Phi_{v}=3.4 \times 10^{-8} \mathrm{G} \mathrm{cm}^{2}$, where $\nu$ is the rotation frequency of the star. The radial dimension of the vortices, approximately equal to the inter-vortex distance, is obtained as follows,

$R_{c}=\frac{1}{\sqrt{\mathcal{D}}}=\frac{0.016}{\sqrt{\nu}} \mathrm{cm}$.

For example, for a pulsar with $P=1 \mathrm{~ms}, R_{c}=2.02 \times 10^{-4} \mathrm{~cm}$. The penetration depth $\lambda$ is given by,

$\lambda=\left(\frac{m_{s}^{*} c^{2}}{4 \pi n_{s}^{*} q_{s}^{* 2}}\right)^{1 / 2}$

where $n_{s}^{*}$ is the number density of the $s s$-pairs. For $s s$-pairs the potential is attractive in the $L=1$ channel implying that the centrifugal barrier in the $p$-state reduces the potential effectively. A simple minded calculation shows that the $s s$-pair density is $\sim 10^{-8} \mathrm{fm}^{-3}$ giving rise to a large value of $\lambda$. Using all these we have,

$B=\frac{\Phi_{v}}{\pi \lambda^{2}} \sim 10^{8} \mathrm{G}$

Evidently, the small value of $B$ is a manifestation of the large $\lambda$.

\section{CONCLUSION}

In this work, we have estimated the minimum magnetic field at which the Ginzburg-Landau free energy of the SQM (with two types of di-quark condensate) is minimized. For suitable QCD parameters this appears to be $\sim 10^{8} \mathrm{G}$. It is mostly due to a large coherence length of the ss-pair $\left(\sim 10^{5} \mathrm{fm}\right)$ since they are in the 
Ray Mandal et al.

$L=1$ state. The centrifugal barrier of higher $L$-state would reduce the $s s$-pair density. This and the large strange quark mass increase the coherence length, $\xi$. It is still much smaller than the electron superconductor coherence length but it is large compared to the relevant QCD scale of $1 \mathrm{fm}$.

Although the magnetic field of $\sim 10^{8} \mathrm{G}$ is astronomically very appealing, given the uncertainties in our parameters and the rough nature of the estimate the actual value could differ by an order of magnitude or so. The important conclusion of our study however is obtaining a lower bound for the magnetic field. Once this value is reached the magnetic field would not spontaneously decay (by ohmic dissipation etc..) to a lower field strength if there is no external perturbation present. Therefore, if the neutron stars in the low mass binary systems do indeed undergo a deconfinement transition to convert into strange stars we should not expect to see any radio pulsar with field values significantly smaller than $\sim 10^{8}$ Gauss.

Whether or not some (or all) of the pulsars are strange stars is an old debate. One of the arguments against the strange stars have been the fact that they would not be able to sustain glitches because the matter content of the hadronic crust of such stars are tiny compared to the magnitude of the glitches observed (see (Madsen 2004) for a recent update on this controversy). The recent observation of a microglitch $\left(\delta P / P \sim 10^{-11}\right)$ in the millisecond pulsar PSR B1821-24 by Cognard \& Backer (2004) lends some credence to our hypothesis that the millisecond pulsars are strange stars. Because a small hadronic crust of a strange star would be just right to give rise to such a micro-glitch.

\section{ACKNOWLEDGMENT}

We would like to thank R. Dey, R. Gopakumar, B. P. Mandal, T. V. Ramakrishnan, S. Ray and D. Shah for helpful discussions. MD, JD, MB acknowledge the hospitality of HRI, Allahabad and SK thanks the astrophysics group of SISSA/ISAS, Trieste for the same. This work has been supported by a DST (Government of India) grant (No. SP/S2/K-03/01).

\section{REFERENCES}

Alcock C., Farhi E., Olinto A., 1986, ApJ, 310, 261

Bailin D., Love A., 1984, Phys. Rep., 107, 325

Baym G., 1988, in Broglia R. A., Schreiffer J. R., ed, Frontiers and Borderlines in Many Particle Physics. Italian Physical Society, Bologna, p. 330

Benvenuto O. G., Horvath J. E., Vucetich H., 1991, Phys. Rev. D, 44, 1321

Benvenuto O. G., Vucetich H., Horvath J. E., 1991, Int. J. Mod. Phys., A6, 4769

Bhattacharya D., 2002, JA\&A, 22, 67

Bombaci I., Thampan A. V., Datta B., 2000, ApJ, 541, L71

Chau H. F., 1997, ApJ, 479, 886

Cognard I., Backer D. C., 2004, ApJ, 612, L125

Datta B., Thampan A. V., Bombaci I., 2000, A\&A, 355, L19

Dey M., Bombaci I., Dey J., Ray S., Samanta B. C., 1998, Phys. Lett. B, 438,123

Gondek-Rosińska D., Bulik T., Zdunik L., Gourgoulhon E., Ray S., Dey J., Dey M., 2000, A\&A, 363, 1005

Haensel P., Zdunik J. L., Schaefer R., 1986, A\&A, 160, 121
Li X.-D., Bombaci I., Dey M., Dey J., van den Heuvel E. P. J., 1999, Phys. Rev. Lett., 83, 3776

Madsen J., 2004, Physical Review Letters, 92, 119002

Olinto A. V., 1987, Phys. Lett. B, 192, 71

Sauls J. A., 1989, in Ögelman H., van den Heuvel E. P. J., ed, Timing Neutron Stars. Kluwer, Dordrecht, p. 457

Sinha M., Dey M., Ray S., Dey J., 2002, MNRAS, 337, 1368

Sinha M., Ray S., Dey M., Dey J., 2005, astro-ph/0504292

Witten E., 1984, Phys. Rev. D, 30, 272

Zhang C., 2004, in IAU Symposium, p. 47

This paper has been typeset from a $\mathrm{T}_{\mathrm{E}} \mathrm{X} / \mathrm{LT}_{\mathrm{E}} \mathrm{X}$ file prepared by the author. 